\title{
Inhibitors of Human Neuraminidase Enzymes Block Transmigration in vitro
}

\author{
Md. Amran Howlader, Tianlin Guo and Christopher W. Cairo* \\ Department of Chemistry, University of Alberta, Edmonton, AB, Canada
}

Cell migration to a site of inflammation is an important step of the immune response. This process is coordinated by cytokines, receptors, and the signal processing machinery of the cell. Many cellular receptors are glycosylated, and their activity can be modulated through changes in glycan structure. Furthermore, glycosylation can be critical to the folding and trafficking of receptors. In this work, we investigated the role of native human neuraminidase enzymes (NEU) in transmigration. We used a cultured T cell line (Jurkat) and a transwell assay with fibronectin (FN) coated wells and cytokines (IL-4 and TNF- $\alpha$ ) as chemoattractants in the bottom chamber. We observed that NEU1, NEU3, and NEU4 were positive regulators of transmigration using an siRNA knockdown. Furthermore, we found that pharmacological inhibition of these enzymes inhibited transmigration. We conclude that human NEU isoenzymes NEU1, NEU3, and NEU4 can act as positive regulators of transmigration and should be investigated as targets for anti-inflammatory strategies.

Edited by:

Chantelle J. Capicciotti, Queen's University, Canada

Reviewed by: Richard Steet, Greenwood Genetic Center, United States Landon John Edgar, University of Toronto, Canada

*Correspondence: Christopher W. Cairo ccairo@ualberta.ca

Specialty section: This article was submitted to Glycoscience,

a section of the journal Frontiers in Molecular Biosciences

Received: 14 December 2021 Accepted: 01 February 2022 Published: 25 February 2022

Citation: Howlader MA, Guo T and Cairo CW (2022) Inhibitors of Human Neuraminidase Enzymes Block Transmigration in vitro.

Front. Mol. Biosci. 9:835757. doi: 10.3389/fmolb.2022.835757
Keywords: neuraminidase, sialidase, sialic acid, cell adhesion, migration, transmigration, chemotaxis

\section{INTRODUCTION}

The migration of leukocytes to a site of inflammation is an important step in the inflammatory cascade (Ley et al., 2007). Leukocytes can become activated upon stimulation by cytokines and engage a series of receptors in peripheral tissue that allow them to home to the site of inflammation (Springer, 1994; Johnston and Butcher, 2002). Importantly, most of these receptors are glycosylated, and changes to their glycan structures may regulate immune function (Rudd et al., 1999; Marth and Grewal, 2008). Many glycan structures are terminated by $\mathrm{N}$-acetyl-neuraminic acid (sialic acid), and the presence of this residue can be critical for protein-glycan recognition. A primary example of this phenomenon is the sialylLewis $\mathrm{x}$ antigen (CD15s). The CD15s antigen acts as a selectin ligand, but loss of the sialic acid from the antigen (forming CD15) prevents selectin binding (Tiemeyer et al., 1991). Thus, regulation of sialyation is critical to such interactions (Gadhoum and Sackstein, 2008). Sialic acid residues are installed by sialyltransferase and removed by neuraminidase (NEU; or sialidase) enzymes (Varki et al., 2015).

There are four isoenzymes of human NEU, NEU1-4, which have distinct subcellular and tissue expression and substrate preferences (Miyagi and Yamaguchi, 2012). The NEU1 enzyme hydrolyzes $\mathrm{N}$-acetyl-neuraminic acids (sialic acids) from proteins, while NEU3 acts primarily on glycolipids. NEU4 can modify both glycoproteins and glycolipids (Wang et al., 2001; Seyrantepe et al., 2004; Sandbhor et al., 2011; Smutova et al., 2014). The human NEU enzymes have a growing list of potential functions in the immune system; some examples include: removal of leukocyte selectin ligands (Gadhoum and Sackstein, 2008), regulation of toll-like receptors (Amith et al., 2010), regulation of cytokine production (Stamatos et al., 2010), modulation of integrin adhesion 
(Howlader et al., 2019), and regulation of LDL uptake by macrophages in atherosclerosis (Demina et al., 2021).

Our understanding of the role of human NEU in the regulation of cell adhesion and migration is only beginning to emerge. In previous studies, we have investigated the influence of human NEU on the migration of adherent cells on a FN substrate through the $\beta 1$-integrin (Howlader et al., 2020). In those studies, we observed distinct roles for isoenzymes: NEU1 was a negative regulator of migration, while NEU3 and NEU4 were positive regulators. We reasoned that a similar difference in NEU function in lymphocytes could have important implications for these enzymes in anti-inflammatory or wound-healing applications. Here, we investigated the influence of human NEU isoenzymes NEU1, NEU3, and NEU4 on $\beta 1$-integrin dependent transmigration in a lymphocyte model (Jurkat T cells). We used siRNA to test the role of native expression of the enzymes, and small molecule inhibitors to test their role pharmacologically. We find that NEU1, NEU3, and NEU4 act as positive regulators of transmigration, suggesting these enzymes could be novel antiinflammatory targets.

\section{MATERIALS AND METHODS}

\section{Materials}

NEU1 siRNA, NEU3 siRNA, and scRNA was procured from Dharmacon, United States (see Supplementary Table S1 for sequences). Lipofectamine 2000 was procured from Thremofisher, United States. Rabbit anti-human $\beta$-actin antibody (ab8227) was procured from Abcam, United States. HRP-conjugated Goat antirabbit and goat anti-mouse antibodies were procured from Biorad, United States. Tumor Necrosis Factor- $\alpha$ (TNF- $\alpha$ ), Interleukin-4 (IL4), Streptavidin-HRP, and mouse anti-human NEU1 antibody was procured from R\&D systems, United States. Mouse anti-human NEU3 antibody (Clone 11B) was procured from MBL international (Woburn, MA, United States). Fibronectin (FN) was procured from Calbiochem, United States. Maackia amurensis (MAL) and Sambucus Nigra (SNA) lectins were procured from bio-World, United States. Jurkat T cells (Clone E6.1) were procured from ATCC (Manassas, VA, United States). Inhibitors CG14600 1, C9-BA-DANA 2, CG22600 3, CZ53100 4, CY16600 5, and DANA 6 were prepared and characterized as previously reported (Zou et al., 2010; Zhang et al., 2013; Guo et al., 2018a; Guo et al., 2018b).

\section{Transfection of Jurkat T cells With siRNA}

Briefly, siRNA $(20 \mu \mathrm{L})$ and lipofectamine $2000(10 \mu \mathrm{L})$ was mixed in serum-free RPMI medium. Then Jurkat T cells $\left(5 \times 10^{6}\right)$ were treated with NEU1 siRNA, NEU3 siRNA, or a scrambled control (scRNA) mixture for $72 \mathrm{~h}$ following the manufacturer's protocol (Thermofisher, United States) without antibiotics. After incubation, cells were collected and lysed using RIPA buffer. Protein concentrations were measured using a BCA assay and equal amounts of protein were loaded in each lane of a gel used for Western blots. Western blots for NEU1 used a mouse antihuman NEU1 antibody (1:5000 dilution); and for NEU3 used mouse anti-human NEU3 antibody (1:2000 dilution). HRPconjugated goat anti-mouse antibody was used as a secondary antibody. The blots were developed using enhanced chemiluminescence reagent (ECL, Biorad, United States) (Supplementary Figures S2-S4). As a secondary check for protein loading, we performed a separate blot for $\beta$-actin. The primary $\beta$-actin antibody was used at 1:5000 dilution, and secondary antibody was used at 1:3000 dilution.

\section{Transwell Migration Assays}

Transwell migration assays were performed following protocols from Justus et al. (2014), with slight modifications for Jurkat T cells. Jurkat T cells $\left(250 \mu \mathrm{L}, 1 \times 10^{4}\right.$ cells well $\left.^{-1}\right)$ were carefully spread on top of a FN-treated $3 \mu \mathrm{m}$ pore upper chamber of a Costar transwell plate (Corning, United States). The upper chamber was maintained with serum-reduced RPMI medium. Inhibitors were applied on both the upper and the lower chambers at the indicated concentration. The bottom chamber was loaded with media containing cytokines (IL-4, $20 \mathrm{ng} / \mathrm{ml}^{-1}$; TNF- $\alpha, 10 \mathrm{ng} \mathrm{ml}^{-1}$ ) and $10 \%$ FBS in RPMI media as a chemoattractant (Leung et al., 1994; Xia et al., 1996). Serumfree RPMI was used in the bottom chamber as a negative control. Cells were allowed to transmigrate for $21 \mathrm{~h}$ at $37^{\circ} \mathrm{C}$ in a humidified incubator with $5 \% \mathrm{CO}_{2}$. Cells were then collected from the bottom chamber and counted using a flow cytometer (BD Accuri C6, BD Biosciences). Normalized transmigration was calculated using the formula: Normalized transmigration, $\operatorname{Tr}=\left(\frac{\mathrm{N} 21}{N_{B}}\right) \times 100$. Where $\mathrm{N}_{21}$ was the number of live cells per $50 \mu \mathrm{L}$ of medium in the bottom chamber after $21 \mathrm{~h}$, and $\mathrm{N}_{\mathrm{B}}$ was the number of live $\mathrm{T}$ cells per $50 \mu \mathrm{L}$ of medium in the bottom chamber of the control well.

\section{Cell Viability Assays}

Viability of cells after treatment was assayed under similar conditions that were used in the transmigration studies. Briefly, wells of a 96 well plate were charged with $100 \mu \mathrm{L}$ of $50 \times 10^{4}$ cells $\mathrm{mL}^{-1}$ and incubated for $18 \mathrm{~h}$ at $37^{\circ} \mathrm{C}$ in a humidified incubator with $5 \% \mathrm{CO}_{2}$. Cells were then treated with the indicated conditions for $21 \mathrm{~h}$. The final concentration of the compounds was the same as that used for transmigration studies. After incubation, $20 \mu \mathrm{L}$ of MTS solution (Promega, United States) was added to each well and incubated for $2 \mathrm{~h}$. The plate was spun at $200 \mathrm{x}$ g for $5 \mathrm{~min}$ to settle the cells on the bottom of the plate. The absorbance of soluble formazan produced by viable cells from metabolism of MTS was measured at $490 \mathrm{~nm}$ using SpectraMax M2 (Molecular Devices, United States) plate reader. For each condition, the experiment was conducted with replicates on different days to consider the intra- and inter-day variabilities. Absorbance for each replicate was normalized to that of an intra-day buffer control.

\section{RESULTS}

\section{NEU1 and NEU3 Expression Levels Were Reduced in Jurkat $T$ cells Using siRNA}

Before investigating the activity of NEU enzyme inhibitors, we sought to determine the role of native NEU expression in transmigration. We selected Jurkat $\mathrm{T}$ cells as a reproducible and stable $\mathrm{T}$ cell line for our experiments. The expression of NEU1, NEU3, and NEU4 has been altered through transfection 


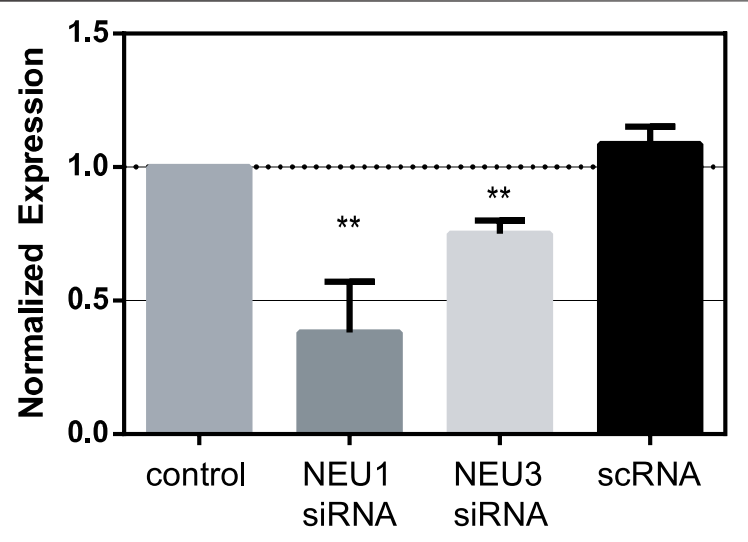

FIGURE 1 | Knockdown of NEU1 and NEU3 expression using siRNA. Jurkat cells were transfected with siRNA for NEU1, NEU3, or with a scrambled control (scRNA). Western blots were used to determine changes in expression of NEU1 and NEU3 (see Supporting Information). Blots were analyzed by densitometry, and the average changes in expression are shown relative to controls. Values were compared to control using a Student's t-test $\left({ }^{\star \star} p<0.01\right)$

of cells with siRNA (Howlader et al., 2020). Using siRNA, we were able to significantly reduce the expression levels of NEU1 by $\sim 60 \%$ and that of NEU3 by $\sim 20 \%$ (Figure 1; Supplementary Figures S2-S4). Unfortunately, we were unable to measure changes in NEU4 expression levels as we were unable to find a suitable antibody for Western blots (Howlader et al., 2020). We used an identical protocol for siRNA knockdown of NEU1, NEU3, and NEU4 to investigate the influence of these enzymes on $\mathrm{T}$ cell transmigration.

\section{Interference with NEU1, NEU3, and NEU4 Expression Reduced Transmigration}

We implemented a transwell assay to investigate the effects of $\mathrm{NEU}$ isoenzyme expression on Jurkat $\mathrm{T}$ cell transmigration (Justus et al., 2014). Cells were seeded into the top chamber of a transwell plate, where the filter was pre-treated with fibronectin (FN). The bottom chamber contained media with serum and inflammatory cytokines as chemoattractants (TNF- $\alpha$ and IL-4) (Leung et al., 1994; Xia et al., 1996). The bottom chamber was loaded with serum- and cytokine-free media for negative controls. Cells were pre-treated with scRNA or siRNA for NEU knockdown of the relevant enzyme before being seeded into the transwell chamber. Once loaded into the chamber, cells were allowed to migrate for $21 \mathrm{~h}$, collected from the bottom chamber and then counted by flow cytometry. We observed that cytokines were required to obtain significant chemotaxis of the cells. Compared to scRNA controls, we observed that siRNA for NEU1, NEU3, and NEU4 significantly inhibited transmigration (Figure 2; Supplementary Table S2). We concluded that NEU1, NEU3, and NEU4 act as positive regulators of transmigration. As noted above, we could not confirm a change in expression level for NEU4; however, these results are consistent with pharmacological inhibition (vide infra) (Howlader et al., 2020).

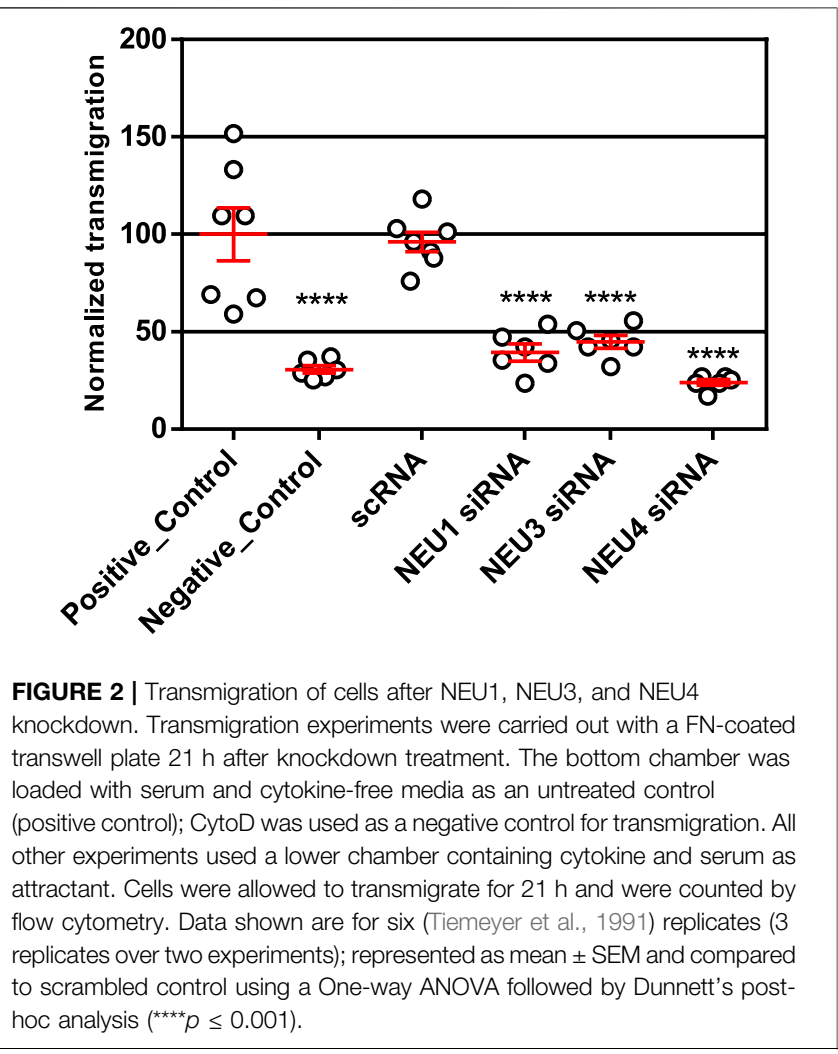

\section{Inhibitors of NEU1, NEU3, and NEU4 Block Transmigration}

Our group (Albohy et al., 2013; Cairo, 2014; Guo et al., 2018a; Guo et al., 2018b), and others (Magesh et al., 2008), have reported the development of selective inhibitors of the human NEU enzymes. Interestingly, widely known inhibitors of viral NEU enzymes are not very active or selective against the human enzymes (Hata et al., 2008; Richards et al., 2018). For this study, we identified a set of inhibitors with variable selectivity for NEU1, NEU3, and NEU4 to investigate for effects on transmigration (Figure 3). Compounds were tested over a concentration range of $1-100 \mu \mathrm{M}$ in transmigration assays using wild-type Jurkat T cells. For these experiments, cytochalasin D (cytoD) was used as a negative control for cell transmigration. CytoD acts by disruption of the actin cytoskeleton by stopping the polymerization of actin monomers (Wakatsuki et al., 2001). Since the structure of the actin cytoskeleton is essential for cell movement, CytoD is a potent inhibitor of cell migration. The transwell assay again used serum and cytokines as chemoattractants in the bottom chamber, and cells were seeded into the top chamber with the indicated concentration of inhibitor (Figure 4).

We tested two inhibitors of NEU1, CG14600 1 and C9-BADANA 2 . Both compounds are reported to have $\sim 30$-fold selectivity for NEU1 (Magesh et al., 2008; Guo et al., 2018a. We observed that both compounds were active as inhibitors of transmigration at the highest concentration tested $(100 \mu \mathrm{M})$. Between these, CG14600 1 treatment showed a more potent response with significant inhibition at concentrations as low as $10 \mu \mathrm{M}$. Compound CY16600 5 has 500- 

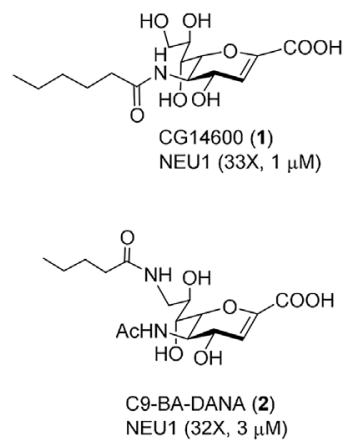
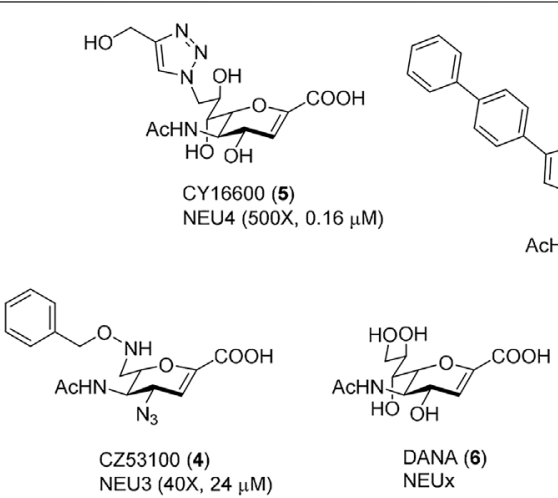<smiles></smiles><smiles></smiles>

FIGURE 3 | Selective human NEU inhibitors used in this study. Inhibitors were selected from previous reports for selectivity against different human NEU isoenzymes. Each compound is shown with a unique identifier and a compound number used in this work. The isoenzyme which the inhibitor targets is given, along with

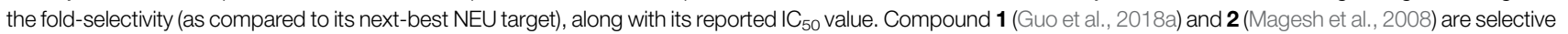
NEU1 inhibitors. Compound $\mathbf{3}$ (Guo et al., 2018b) and $\mathbf{4}$ (Zhang et al., 2013) are selective NEU3 inhibitors. Compound $\mathbf{5}$ (Albohy et al., 2013 ) is a selective NEU4 inhibitor. Compound $\mathbf{6}$ is a general inhibitor with activity for all human NEU isoenzymes (Richards et al., 2018).

fold selectivity for NEU4 and is the most potent human NEU inhibitor reported to date (Albohy et al., 2013). When tested in the transwell assay, we observed a dose-dependent inhibition of migration with significant effects at $1-100 \mu \mathrm{M}$. We tested two inhibitors of NEU3, CZ53100 4 and CG22600 3, which differ in both their potency and selectivity (Figure 3; Supplementary Table S3) (Zhang et al., 2013; Guo et al., 2018b). Both the compound CZ53100 4 and CG22600 3 showed significant inhibition of migration at the $10-100 \mu \mathrm{M}$ concentration. Although CG22600 3 showed inhibition at concentrations as low as $10 \mu \mathrm{M}$, the highest concentration showed more than $50 \%$ inhibition of migration. Finally, to provide a point of comparison, we tested a nonspecific inhibitor of NEU enzymes, DANA 6. As an inhibitor, DANA has activity against all four human isoenzymes with some preference for NEU3 and NEU4 (Richards et al., 2018). We observed moderate inhibition of migration for DANA at $50 \mu \mathrm{M}$; however, higher concentrations did not show significant activity. We speculate that the mixed activity of DANA is a result of its non-specific effects on different NEU isoenzymes (Howlader et al., 2020).

\section{Inhibitors of Human NEU Enzymes did Not Reduce the Viability of cells}

A possible explanation for reduced transmigration of cells in the presence of inhibitors is toxicity of the compounds. To test this hypothesis, we evaluated the cytotoxicity of compounds CG14600 1, CG22600 3, CY16600 5, and DANA 6 in Jurkat T cells (Figure 5; Supplementary Table S4). Compounds were tested under identical conditions used in cell migration experiments, with the highest concentration used for each compound. We did not observe any significant cytotoxicity for the inhibitors tested. Compounds CG14600 1 and CY16600 5 showed slightly increased viability. Treatment of cells with the cytokines used as chemoattractants (IL-4 and TNF- $\alpha$ ) had no effect on $\mathrm{T}$ cell viability (Supplementary Figure S1; Supplementary Table S5). From these experiments, we concluded that the cytotoxicity of the inhibitors was not responsible for the observed effects on transmigration.

\section{DISCUSSION}

In this study, we examined the role of native human NEU isoenzymes in transmigration. We show that reduced expression of NEU1, NEU3, and NEU4 attenuated transmigration, suggesting these enzymes could be considered positive regulators of transmigration. We then tested the effects of specific inhibitors of human NEU isoenzymes on transmigration on FN-treated transwell inserts using TNF- $\alpha$ and IL-4 as chemoattractant. In agreement with our siRNA results, we observed that selective inhibitors of human NEU1, NEU3, and NEU4 could inhibit transmigration. Among these, CG22600 3 showed the greatest potency. We also tested the pan-selective NEU inhibitor, DANA 6, which was found to be a weak inhibitor of transmigration. Finally, we confirmed that the activity of our compounds was not due to cytotoxicity. Together, our results establish a role for human neuraminidase enzymes in the regulation of cell migration and may suggest these enzymes as targets for the development of anti-inflammatory therapeutics.

The glycosylation of extracellular receptors plays a critical role in cell migration and adhesion. Here, we examined the influence of human NEU enzyme activity on a lymphocyte migration model dependent on $\beta 1$-integrin-FN interactions. Glycosylation of integrin receptors is known to affect adhesion (Bellis, 2004; Schultz et al., 2012), and we sought to investigate if NEU enzymes could regulate transmigration in lymphocytes. We have previously found that NEU3 and NEU4 can modify $\beta 1$ integrin in vitro, and that NEU activity may affect integrin endocytosis (Howlader et al., 2020). Our siRNA experiments (Figure 2) establish that NEU1 and NEU3 act as positive regulators of transmigration in our model. The data are also consistent with NEU4 acting as a positive regulator, but we could not confirm changes in NEU4 expression. We also caution that our results used only one cell type (Jurkat $\mathrm{T}$ cells) and chemoattractant with an in vitro model. Future work is planned to investigate if these effects are consistent in primary leukocytes. 

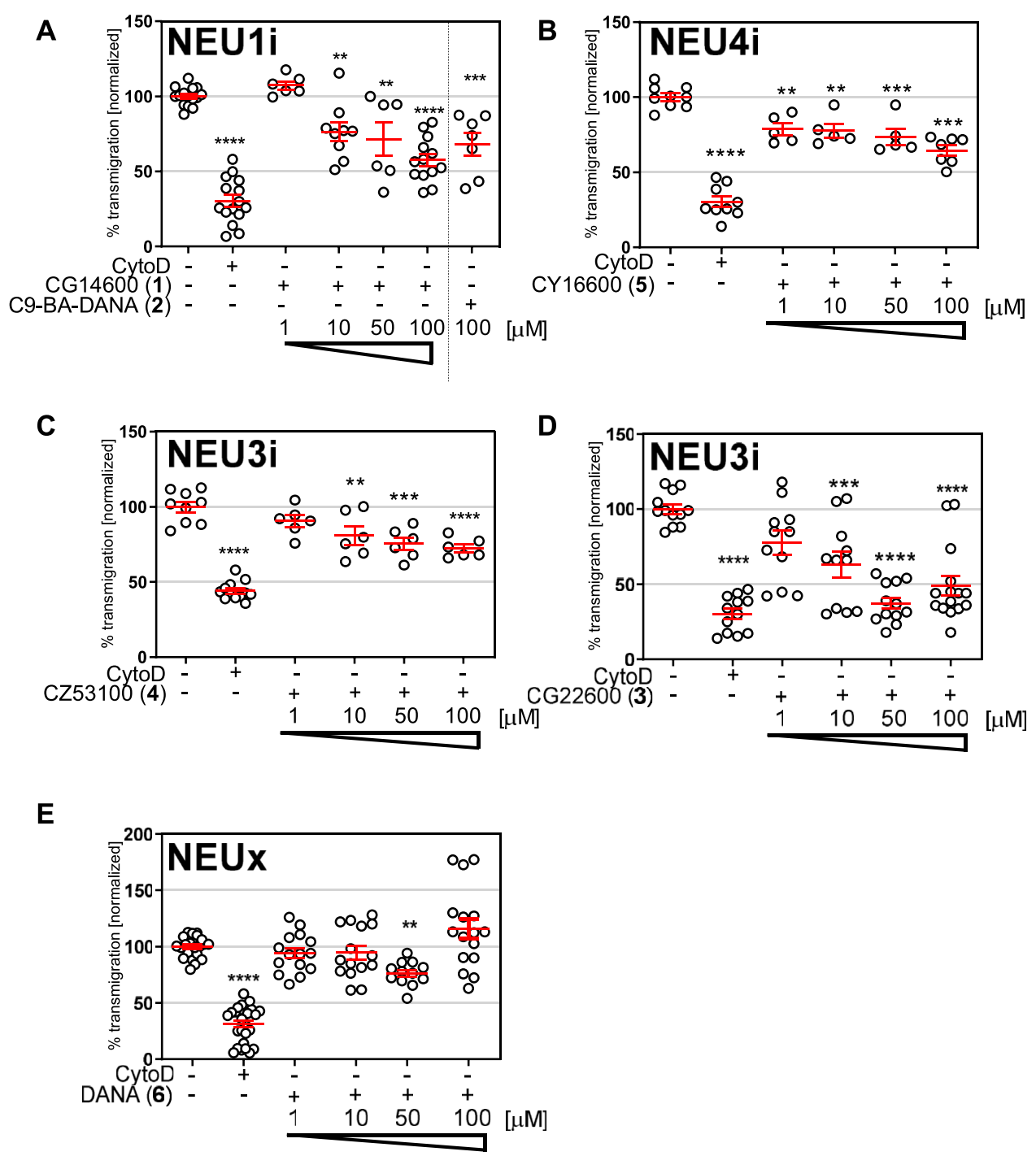

FIGURE 4 | Transmigration after treatment with NEU inhibitors. Transmigration experiments were carried out with a FN-coated transwell plate. The bottom chamber was loaded with serum and cytokine-free media as an untreated control; CytoD was used as a negative control for transmigration. All other experiments used a lower chamber containing cytokine and serum as attractant. Cells were loaded in the top well with the indicated concentration of inhibitors for (A) NEU1, (B) NEU4, (C,D) NEU3, or (E) non-specific inhibitor DANA 6. Cells were allowed to transmigrate for $21 \mathrm{~h}$ and were counted by flow cytometry. Data shown for inhibitors include six technical replicates and two biological replicates; and are represented as mean \pm SEM and compared to control using one-way ANOVA followed by Dunnett's post-hoc analysis $\left({ }^{\star \star \star \star} p \leq 0.001 ;{ }^{\star \star \star} p<0.005 ;{ }^{\star \star} p<0.01\right)$.

Although inhibitors of NEU enzymes are well known in antiviral therapy, clinically approved viral NEU inhibitors have limited activity and specificity against human NEU (Hata et al., 2008; Richards et al., 2018). The presence of four isoenzymes of NEU in humans presents a possibility for off-target effects with non-specific NEU inhibitors. These factors can complicate the interpretation of results for human NEU when using DANA $\mathbf{6}$, oseltamivir, zanamivir, or other antivirals. In recent years, inhibitors selective for individual human NEU isoenzymes have been developed in recognition of this limitation (Magesh et al., 2008; Zhang et al., 2013; Albohy et al., 2013; Cairo, 2014; Guo et al., 2018a; Guo et al., 2018b). These compounds allowed us to interrogate NEU1, NEU3, and NEU4 pharmacologically in transmigration (Figure 4). We found that inhibitors of NEU1, NEU3, and NEU4 all acted as inhibitors of transmigration. The most potent of these were compounds specific for NEU3. In previous studies, we have observed that inhibitors that target different isoenzymes can compete with each other, resulting in masking the role of individual NEU isoenzymes (Howlader et al., 2020). This effect may explain why DANA 6 showed only marginal activity as an inhibitor in our transmigration assays. We also cannot rule out whether differences in the physical properties of these compounds or their recognition by transporters could play a role in these observed differences.

Sialoglycoproteins and glycolipids play a variety of roles in cell biology, and the regulation of sialylation may influence 


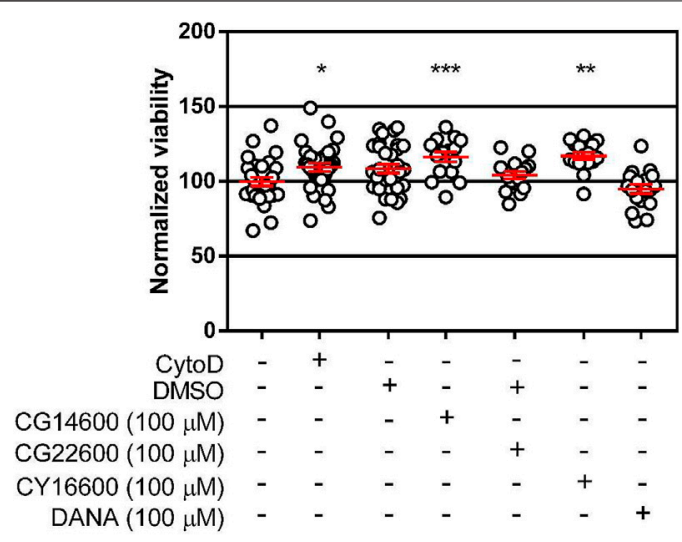

FIGURE 5 | Viability of cells after treatment with inhibitors. Viability of cells was tested after treatment with the highest concentrations of inhibitors used for transmigration studies. Jurkat T cells $\left(5 \times 10^{4}\right.$ cells) were incubated in each well of a clear 96-well plate with the indicated inhibitors for $21 \mathrm{~h}$. After incubation, MTS was used to determine cell viability after $1 \mathrm{~h}$ incubation. Data are plotted as the mean \pm SEM and compared to control using a Dunnett's $t$-test $\left({ }^{\star} p \leq 0.05 ;{ }^{* *} p \leq 0.01 ;{ }^{* *} p \leq 0.005\right)$.

recognition and signaling processes. Sialosides participate in regulating $\mathrm{T}$ cell activation, and targeted degradation of sialosides is emerging as a strategy for immunotherapy (Gray et al., 2020; Edgar et al., 2021). Sialic acids have been found to mediate a large number of glycoprotein interactions, including adhesion receptors such as the $\beta 1$-integrin, a receptor for $\mathrm{FN}$ (Johansson et al., 1997; Schultz et al., 2012; Xie et al., 2021). In cancers that overexpress NEU1 the enzyme has been found to act as a negative regulator of migration through disruption of $\beta 1$-and $\beta 4$-integrin interactions (Uemura et al., 2009; Zhou et al., 2020). In other cancer models, NEU1 has been found to be a positive regulator of cell migration (Hou et al., 2016; Ren et al., 2016). NEU1 has also been found to be a positive regulator of lymphocyte infiltration in pulmonary fibrosis models (Luzina et al., 2016; Luzina et al., 2020). Similarly, NEU3 has been found to act as a positive regulator of $\beta 1$-integrin-mediated cell migration in renal cancer cells (Tringali et al., 2012). Our transmigration model found that all NEU isoenzymes tested acted as positive regulators (NEU1, NEU3, and NEU4). It is likely that extracellular matrix glycoproteins and specific sialoglycoconjugates are critical to the function of NEU enzymes during cell migration in vivo. We previously observed that NanI and NEU3 can alter sialylation of LFA-1 (a $\beta 2$-integrin) in vitro (Howlader et al., 2019), and that NEU enzymes and inhibitors can alter the endocytosis of integrins (Howlader et al., 2019; Howlader et al., 2020). In experiments examining cell migration on a FN-coated surface, we found that pretreatment of FN with NEU did not affect rates of migration (Jia et al., 2016). Thus, we speculate that NEU enzyme activity regulates cell migration through changes to sialoglycoconjugates on the cell surface, such as integrins or glycolipids.

Indeed, NEU isoenzymes have context-dependent effects on cell adhesion, varying by cell type and receptor system. Using an in vitro model of adherent cell migration with a FN substrate, we previously reported that NEU3 and NEU4 act as positive regulators of migration, while NEU1 acted as a negative regulator (in PC-3 and MBA-MD-231 cells) (Howlader et al., 2020). In the case of NEU3 and NEU4, these enzymes have activity for glycolipid substrates which may provide a mechanism for their effects (Jia et al., 2016). Furthermore, we have observed that NEU3 acts as a negative regulator of $\beta 2$-integrin-mediated static adhesion while acting as a positive regulator of $\beta 1$-integrin-mediated homotypic aggregation in lymphocytes (Howlader et al., 2019). We propose that using isoenzyme-selective inhibitors of NEU provides new opportunities for understanding the complex role of sialoglycoconjugates and the enzymes that modify them in inflammation and cell migration.

Using an in vitro model of $\mathrm{T}$ cell transmigration, we observed that native activity of NEU1 and NEU3 act as positive regulators. Our data support that NEU4 also acts as a positive regulator in this model, but we could not confirm expression levels for this isoenzyme. Treatment of cells with selective inhibitors of human $\mathrm{NEU}$ isoenzymes allowed us to confirm these effects in the same assay. Small molecule inhibitors selective for NEU1, NEU3, and NEU4 all acted as inhibitors of transmigration. Compounds with mixed selectivity, such as DANA 6, showed less consistent inhibition with reduced potency as compared to selective inhibitors. The activity of selective human NEU inhibitors should be investigated in whole animal models and with different cell types to establish the role of NEU enzymes in inflammation.

\section{DATA AVAILABILITY STATEMENT}

The original contributions presented in the study are included in the article/Supplementary Material, further inquiries can be directed to the corresponding author.

\section{AUTHOR CONTRIBUTIONS}

MH designed experiments, performed experiments, analyzed data, and wrote the manuscript; TG prepared reagents and analyzed data; CC designed experiments, analyzed data, and wrote the manuscript.

\section{FUNDING}

This work was supported by grants from the Canadian Glycomics Network (GlycoNet, ID-01 and CD-2) and the Natural Sciences and Engineering Research Council of Canada (NSERC RGPIN2020-04371).

\section{SUPPLEMENTARY MATERIAL}

The Supplementary Material for this article can be found online at: https://www.frontiersin.org/articles/10.3389/fmolb.2022.835757/ full\#supplementary-material 


\section{REFERENCES}

A. Varki, R. D. Cummings, J. D. Esko, P. Stanley, G. W. Hart, M. Aebi, A. G. Darvill, T. Kinoshita, N. H. Packer, J. H. Prestegard, et al. (2015). "Essentials of Glycobiology," Cold Spring Harbor. 3rd ed. ((NY): Cold Spring Harbor Laboratory Press).

Albohy, A., Zhang, Y., Smutova, V., Pshezhetsky, A. V., and Cairo, C. W. (2013). Identification of Selective Nanomolar Inhibitors of the Human Neuraminidase, NEU4. ACS Med. Chem. Lett. 4, 532-537. doi:10.1021/ml400080t

Amith, S. R., Jayanth, P., Franchuk, S., Finlay, T., Seyrantepe, V., Beyaert, R., et al. (2010). Neul Desialylation of Sialyl $\alpha$-2,3-linked $\beta$-galactosyl Residues of TOLL-like Receptor 4 Is Essential for Receptor Activation and Cellular Signaling. Cell Signal. 22, 314-324. doi:10.1016/j.cellsig.2009.09.038

Bellis, S. L. (2004). Variant Glycosylation: an Underappreciated Regulatory Mechanism for $\beta 1$ Integrins. Biochim. Biophys. Acta (Bba) - Biomembranes 1663, 52-60. doi:10.1016/j.bbamem.2004.03.012

Cairo, C. W. (2014). Inhibitors of the Human Neuraminidase Enzymes. Med. Chem. Commun. 5, 1067-1074. doi:10.1039/C4MD00089G

Demina, E. P., Smutova, V., Pan, X., Fougerat, A., Guo, T., Zou, C., et al. (2021). Neuraminidases 1 and 3 Trigger Atherosclerosis by Desialylating Low-Density Lipoproteins and Increasing Their Uptake by Macrophages. JAHA 10, e 018756. doi:10.1161/JAHA.120.018756

Edgar, L. J., Thompson, A. J., Vartabedian, V. F., Kikuchi, C., Woehl, J. L., Teijaro, J. R., et al. (2021). Sialic Acid Ligands of CD28 Suppress Costimulation of T Cells. ACS Cent. Sci. 7, 1508-1515. doi:10.1021/acscentsci.1c00525

Gadhoum, S. Z., and Sackstein, R. (2008). CD15 Expression in Human Myeloid Cell Differentiation Is Regulated by Sialidase Activity. Nat. Chem. Biol. 4, 751-757. doi:10.1038/nchembio.116

Gray, M. A., Stanczak, M. A., Mantuano, N. R., Xiao, H., Pijnenborg, J. F. A., Malaker, S. A., et al. (2020). Targeted Glycan Degradation Potentiates the Anticancer Immune Response In Vivo. Nat. Chem. Biol. 16, 1376-1384. doi:10. 1038/s41589-020-0622-x

Guo, T., Dätwyler, P., Demina, E., Richards, M. R., Ge, P., Zou, C., et al. (2018). Selective Inhibitors of Human Neuraminidase 3. J. Med. Chem. 61, 1990-2008. doi:10.1021/acs.jmedchem.7b01574

Guo, T., Héon-Roberts, R., Zou, C., Zheng, R., Pshezhetsky, A. V., and Cairo, C. W. (2018). Selective Inhibitors of Human Neuraminidase 1 (NEU1). J. Med. Chem. 61, 11261-11279. doi:10.1021/acs.jmedchem.8b01411

Hata, K., Koseki, K., Yamaguchi, K., Moriya, S., Suzuki, Y., Yingsakmongkon, S., et al. (2008). Limited Inhibitory Effects of Oseltamivir and Zanamivir on Human Sialidases. Antimicrob. Agents Chemother. 52, 3484-3491. doi:10. 1128/AAC.00344-08

Hou, G., Liu, G., Yang, Y., Li, Y., Yuan, S., Zhao, L., et al. (2016). Neuraminidase 1 (NEU1) Promotes Proliferation and Migration as a Diagnostic and Prognostic Biomarker of Hepatocellular Carcinoma. Oncotarget 7, 64957-64966. doi:10. 18632/oncotarget.11778

Howlader, M. A., Guo, T., Chakraberty, R., and Cairo, C. W. (2020). IsoenzymeSelective Inhibitors of Human Neuraminidases Reveal Distinct Effects on Cell Migration. ACS Chem. Biol. 15, 1328-1339. doi:10.1021/acschembio.9b00975

Howlader, M. A., Li, C., Zou, C., Chakraberty, R., Ebesoh, N., and Cairo, C. W. (2019). Neuraminidase-3 Is a Negative Regulator of LFA-1 Adhesion. Front. Chem. 7, 7. doi:10.3389/fchem.2019.00791

Jia, F., Howlader, M. A., and Cairo, C. W. (2016). Integrin-mediated Cell Migration Is Blocked by Inhibitors of Human Neuraminidase. Biochim. Biophys. Acta (Bba) - Mol. Cel Biol. Lipids 1861, 1170-1179. doi:10.1016/j.bbalip.2016.06.013

Johansson, S., Svineng, G., Wennerberg, K., Armulik, A., and Lohikangas, L. (1997). Fibronectin-integrin Interactions. Front. Biosci. 2, d126-146. doi:10. 2741/a178

Johnston, B., and Butcher, E. C. (2002). Chemokines in Rapid Leukocyte Adhesion Triggering and Migration. Semin. Immunol. 14, 83-92. doi:10.1006/smim.2001. 0345

Justus, C. R., Leffler, N., Ruiz-Echevarria, M., and Yang, L. V. (2014). In Vitro cell Migration and Invasion Assays. J. Vis. Exp. Jove.

Leung, K. N., Mak, N. K., Fung, M. C., and Hapel, A. J. (1994). Synergistic Effect of IL-4 and TNF-Alpha in the Induction of Monocytic Differentiation of a Mouse Myeloid Leukaemic Cell Line (WEHI-3B JCS). Immunology 81, 65-72.
Ley, K., Laudanna, C., Cybulsky, M. I., and Nourshargh, S. (2007). Getting to the Site of Inflammation: the Leukocyte Adhesion cascade Updated. Nat. Rev. Immunol. 7, 678-689. doi:10.1038/nri2156

Luzina, I. G., Lillehoj, E. P., Lockatell, V., Hyun, S. W., Lugkey, K. N., Imamura, A., et al. (2020). Therapeutic Effect of Neuraminidase-1-Selective Inhibition in Mouse Models of Bleomycin-Induced Pulmonary Inflammation and Fibrosis. J. Pharmacol. Exp. Ther. 376, 136-146. doi:10.1124/jpet.120.000223

Luzina, I. G., Lockatell, V., Hyun, S. W., Kopach, P., Kang, P. H., Noor, Z., et al. (2016). Elevated Expression of NEU1 Sialidase in Idiopathic Pulmonary Fibrosis Provokes Pulmonary Collagen Deposition, Lymphocytosis, and Fibrosis. Am. J. Physiology-Lung Cell Mol. Physiol. 310, L940-L954. doi:10. 1152/ajplung.00346.2015

Magesh, S., Moriya, S., Suzuki, T., Miyagi, T., Ishida, H., and Kiso, M. (2008). Design, Synthesis, and Biological Evaluation of Human Sialidase Inhibitors. Part 1: Selective Inhibitors of Lysosomal Sialidase (NEU1). Bioorg. Med. Chem. Lett. 18, 532-537. doi:10.1016/j.bmcl.2007.11.084

Marth, J. D., and Grewal, P. K. (2008). Mammalian Glycosylation in Immunity. Nat. Rev. Immunol. 8, 874-887. doi:10.1038/nri2417

Miyagi, T., and Yamaguchi, K. (2012). Mammalian Sialidases: Physiological and Pathological Roles in Cellular Functions. Glycobiology 22, 880-896. doi:10. 1093/glycob/cws057

Ren, L.-r., Zhang, L.-p., Huang, S.-y., Zhu, Y.-f., Li, W.-j., Fang, S. Y., et al. (2016). Effects of Sialidase NEU1 siRNA on Proliferation, Apoptosis, and Invasion in Human Ovarian Cancer. Mol. Cel Biochem 411, 213-219. doi:10.1007/s11010 015-2583-z

Richards, M. R., Guo, T., Hunter, C. D., and Cairo, C. W. (2018). Molecular Dynamics Simulations of Viral Neuraminidase Inhibitors with the Human Neuraminidase Enzymes: Insights into Isoenzyme Selectivity. Bioorg. Med. Chem. 26, 5349-5358. doi:10.1016/j.bmc.2018.05.035

Rudd, P. M., Wormald, M. R., Stanfield, R. L., Huang, M., Mattsson, N., Speir, J. A., et al. (1999). Roles for Glycosylation of Cell Surface Receptors Involved in Cellular Immune Recognition. J. Mol. Biol. 293, 351-366. doi:10.1006/jmbi. 1999.3104

Sandbhor, M. S., Soya, N., Albohy, A., Zheng, R. B., Cartmell, J., Bundle, D. R., et al. (2011). Substrate Recognition of the Membrane-Associated Sialidase NEU3 Requires a Hydrophobic Aglycone. Biochemistry 50, 6753-6762. doi:10.1021/ bi200449j

Schultz, M. J., Swindall, A. F., and Bellis, S. L. (2012). Regulation of the Metastatic Cell Phenotype by Sialylated Glycans. Cancer Metastasis Rev. 31, 501-518. doi:10.1007/s10555-012-9359-7

Seyrantepe, V., Landry, K., Trudel, S., Hassan, J. A., Morales, C. R., and Pshezhetsky, A. V. (2004). Neu4, a Novel Human Lysosomal Lumen Sialidase, Confers normal Phenotype to Sialidosis and Galactosialidosis Cells. J. Biol. Chem. 279, 37021-37029. doi:10.1074/jbc.m404531200

Smutova, V., Albohy, A., Pan, X., Korchagina, E., Miyagi, T., Bovin, N., et al. (2014). Structural Basis for Substrate Specificity of Mammalian Neuraminidases. PLoS ONE 9, e106320. doi:10.1371/journal.pone.0106320

Springer, T. A. (1994). Traffic Signals for Lymphocyte Recirculation and Leukocyte Emigration: the Multistep Paradigm. Cell 76, 301-314. doi:10.1016/00928674(94)90337-9

Stamatos, N. M., Carubelli, I., van de Vlekkert, D., Bonten, E. J., Papini, N., Feng, C., et al. (2010). LPS-induced Cytokine Production in Human Dendritic Cells Is Regulated by Sialidase Activity. J. Leukoc. Biol. 88, 1227-1239. doi:10.1189/jlb. 1209776

Tiemeyer, M., Swiedler, S. J., Ishihara, M., Moreland, M., Schweingruber, H., Hirtzer, P., et al. (1991). Carbohydrate Ligands for Endothelial-Leukocyte Adhesion Molecule 1. Proc. Natl. Acad. Sci. 88, 1138-1142. doi:10.1073/ pnas.88.4.1138

Tringali, C., Lupo, B., Silvestri, I., Papini, N., Anastasia, L., Tettamanti, G., et al. (2012). The Plasma Membrane Sialidase NEU3 Regulates the Malignancy of Renal Carcinoma Cells by Controlling $\beta 1$ Integrin Internalization and Recycling. J. Biol. Chem. 287, 42835-42845. doi:10. 1074/jbc.M112.407718

Uemura, T., Shiozaki, K., Yamaguchi, K., Miyazaki, S., Satomi, S., Kato, K., et al. (2009). Contribution of Sialidase NEU1 to Suppression of Metastasis of Human colon Cancer Cells through Desialylation of Integrin $\beta 4$. Oncogene 28, 1218-1229. doi:10.1038/onc.2008.471 
Wakatsuki, T., Schwab, B., Thompson, N. C., and Elson, E. L. (2001). Effects of Cytochalasin D and Latrunculin B on Mechanical Properties of Cells. J. Cel Sci 114, 1025-1036. doi:10.1242/jcs.114.5.1025

Wang, Y., Yamaguchi, K., Shimada, Y., Zhao, X., and Miyagi, T. (2001). Sitedirected Mutagenesis of Human Membrane-Associated Ganglioside Sialidase. Eur. J. Biochem. 268, 2201-2208. doi:10.1046/j.1432-1327.2001.02069.x

Xia, M., Gaufo, G. O., Wang, Q., Sreedharan, S. P., and Goetzl, E. J. (1996). Transduction of Specific Inhibition of HuT 78 Human T Cell Chemotaxis by Type I Vasoactive Intestinal Peptide Receptors. J. Immunol. 157, 1132-1138.

Xie, Y., Chen, S., Li, Q., Sheng, Y., Alvarez, M. R., Reyes, J., et al. (2021). Glycanprotein Cross-Linking Mass Spectrometry Reveals Sialic Acid-Mediated Protein Networks on Cell Surfaces. Chem. Sci. 12, 8767-8777. doi:10.1039/ D1SC00814E

Zhang, Y., Albohy, A., Zou, Y., Smutova, V., Pshezhetsky, A. V., and Cairo, C. W. (2013). Identification of Selective Inhibitors for Human Neuraminidase Isoenzymes Using C4,C7-Modified 2-Deoxy-2,3-Didehydro-N-Acetylneuraminic Acid (DANA) Analogues. J. Med. Chem. 56, 2948-2958. doi:10.1021/jm301892f

Zhou, X., Zhai, Y., Liu, C., Yang, G., Guo, J., Li, G., et al. (2020). Sialidase NEU1 Suppresses Progression of Human Bladder Cancer Cells by Inhibiting Fibronectin-Integrin a5 11 Interaction and Akt Signaling Pathway. Cell Commun Signal 18, 44. doi:10.1186/s12964-019-0500-x
Zou, Y., Albohy, A., Sandbhor, M., and Cairo, C. W. (2010). Inhibition of Human Neuraminidase 3 (NEU3) by C9-Triazole Derivatives of 2,3-Didehydro-NAcetyl-Neuraminic Acid. Bioorg. Med. Chem. Lett. 20, 7529-7533. doi:10.1016/ j.bmcl.2010.09.111

Conflict of Interest: CC, TG, and MH are co-inventors on patents describing inhibitors of NEU and applications thereof.

Publisher's Note: All claims expressed in this article are solely those of the authors and do not necessarily represent those of their affiliated organizations, or those of the publisher, the editors and the reviewers. Any product that may be evaluated in this article, or claim that may be made by its manufacturer, is not guaranteed or endorsed by the publisher.

Copyright (c) 2022 Howlader, Guo and Cairo. This is an open-access article distributed under the terms of the Creative Commons Attribution License (CC $B Y)$. The use, distribution or reproduction in other forums is permitted, provided the original author(s) and the copyright owner(s) are credited and that the original publication in this journal is cited, in accordance with accepted academic practice. No use, distribution or reproduction is permitted which does not comply with these terms. 